Check for updates

Cite this: Chem. Sci., 2019, 10, 2067

๑ All publication charges for this article have been paid for by the Royal Society of Chemistry

Received 28th June 2018

Accepted 11th December 2018

DOI: $10.1039 / \mathrm{c} 8 \mathrm{sc} 02847 \mathrm{~h}$

rsc.li/chemical-science

\section{A non-sacrificial method for the quantification of poly(ethylene glycol) grafting density on gold nanoparticles for applications in nanomedicine $\uparrow$}

\author{
Jun Lu, ${ }^{a}$ Yao Xue, ${ }^{a}$ Rui Shi, (DD ${ }^{b}$ Jing Kang, ${ }^{a}$ Chao-Yang Zhao, ${ }^{a}$ Ning-Ning Zhang, ${ }^{a}$ \\ Chun-Yu Wang, (D) ab Zhong-Yuan Lu (D) *ab and Kun Liu (D) *a
}

\section{Introduction}

The extraordinary surface plasmon resonance property of gold nanoparticles (GNPs) and their proven ability to transform absorbed light into thermal energy make them promising candidates for a wide range of biological applications, such as bioimaging, medical diagnostics, and photothermal treatment for diseases. ${ }^{1-4}$ However, in vivo studies have shown that most administered GNPs are rapidly sequestered from the bloodstream by the mononuclear phagocyte system (MPS), leading to long-term accumulation of GNPs in the liver and spleen, and inadequate delivery and/or targeting of GNPs to the diseased tissues. $^{5-8}$ The process of sequestration is triggered by opsonization of GNPs, which involves non-specific adsorption of plasma proteins onto the nanoparticle (NP) surface to form the so-called "protein corona", ${ }^{9,10}$ The "protein corona" alters the size, aggregation state, and interfacial properties of GNPs, giving them a "biological identity" that governs the interactions

${ }^{a}$ State Key Laboratory of Supramolecular Structure and Materials, College of Chemistry, Jilin University, Changchun 130012, P. R. China. E-mail: kliu@jlu.edu.cn ${ }^{b}$ Institute of Theoretical Chemistry, Jilin University, Changchun 130023, P. R. China. E-mail: luzhy@jlu.edu.cn

$\dagger$ Electronic supplementary information (ESI) available. See DOI: $10.1039 / \mathrm{c} 8 \mathrm{sc} 02847 \mathrm{~h}$ with biomolecules and biological barriers while affecting the physiological immune response. ${ }^{11,12}$

To suppress the sequestration by MPS, it is crucial to prevent non-specific protein adsorption onto the surface of GNPs. For this purpose, the surface of GNPs needs to be modified with charge-neutral, highly hydrophilic "anti-fouling" polymers, which can resist adsorption of biomolecules, cells, and microorganisms when NPs are injected into living organisms. ${ }^{13-15}$ Poly(ethylene glycol) (PEG) is the most commonly used "antifouling" polymer to modify GNPs in order to suppress nonspecific protein attachment, prolong NP circulation time and improve delivery efficiency to diseased tissues. The grafting density of thiol-terminated PEG (HS-PEG) on the GNP surface $(\sigma)$ is one of the most important parameters in determining the interaction of NPs with proteins, its subsequent sequestration from the bloodstream by the MPS, and the eventual delivery efficiency to tumours. ${ }^{16-19}$ However, studies on optimization of $\sigma$ are relatively rare ${ }^{20,21}$ as the majority of in vivo studies use GNPs saturated with PEG chains but do not characterize or report $\sigma$. The lack of characterization is largely due to a lack of practical methods.

Several types of techniques have been utilized to quantify $\sigma$ of PEG on the surface of NPs. Thermogravimetric analysis (TGA) is the most widely used tool to estimate $\sigma$ of PEG on GNPs; ${ }^{22-25}$ this technique normally requires a large amount of GNPs and multiple centrifugation steps to remove unbound PEG chains, 
which may cause bound PEG to detach from the NP surface and result in an underestimation of $\sigma$. Additionally, TGA is a sacrificial method and impractical as a routine analytical approach for biomedical studies. Another commonly used strategy to characterize $\sigma$ is based on the quantification of thiol end-group of unbound PEG by a stoichiometric reaction with 5,5'dithiobis(2-nitrobenzoic acid). ${ }^{21}$ However, this method cannot quantify the concentration of disulphide bonds which are unavoidably formed in solution, leading to an overestimate of $\sigma$. Other approaches for characterizing $\sigma$ include fluorescence ${ }^{26}$ and $\operatorname{Raman}^{27}$ spectroscopies (limited to HS-PEG molecules containing amino or Raman active end-groups) and other sacrificial methods such as inductively coupled plasma mass spectrometry (ICP-MS), ${ }^{28}$ total organic carbon (TOC) analysis, ${ }^{29}$ or X-ray photoelectron spectroscopy (XPS). ${ }^{30}$ On the other hand, one-dimensional proton nuclear magnetic resonance $\left({ }^{1} \mathrm{H} \mathrm{NMR}\right)$ spectroscopy is an easy-to-use, fast, and non-sacrificed quantification method to quantify $\sigma$. It can also be applied to analyse the ligand chemistry and monitor the grafting process in situ. However, using NMR to quantify $\sigma$ and to simultaneously study the grafting process is quite rare. ${ }^{31,32}$ As such, a simple, robust, and universal NMR analytical method to accurately quantify $\sigma$ in situ is highly desirable.

Herein, we report a facile and non-sacrificial ${ }^{1} \mathrm{H}$ NMR analytical approach for the quantitative characterization of $\sigma$ of HS-PEG on GNPs. We propose using a multi-Lorentziansplitting algorithm based on the Akaike's information criterion (AIC), Bayesian information criterion (BIC) and maximum likelihood estimation in order to distinguish the NMR signal of free PEG from that of grafted PEG, which allows monitoring of the grafting process in situ. We demonstrate that this is an efficient method for studying the grafting rates and grafting densities for GNPs with varying dimensions, PEG with a range of molecular weights, and different types of original capping ligands on GNPs.

\section{Results and discussion}

\section{Quantification of grafting density by multi-Lorentzian- splitting algorithm}

${ }^{1} \mathrm{H}$ NMR spectroscopy was used to monitor the grafting-to process of HS-PEG on $13 \mathrm{~nm}$ GNPs with sodium 2,2-dimethylisotope-2-silapentane-5-sulfonate (DSS) as an external standard. Fig. 1 shows the temporal evolution of the ${ }^{1} \mathrm{H}$ NMR spectra when excess HS-PEG $\left(M_{\mathrm{w}}\right.$ of $\left.5.0 \mathrm{~kg} \mathrm{~mol}^{-1}, 0.50 \mathrm{mg} \mathrm{mL}^{-1}\right)$ was grafted onto the surface of citrate-covered GNPs (78 nM) (Fig. S1 $\dagger$ ). Before grafting, HS-PEG in $\mathrm{D}_{2} \mathrm{O}$ exhibited a sharp peak at $3.70 \mathrm{ppm}$ with a full width at half maximum (FWHM) of $0.00430 \mathrm{ppm}$. Three minutes after the GNPs and HS-PEG were mixed in $\mathrm{D}_{2} \mathrm{O}$, the intensity of the peak was dropped dramatically to about half of its initial value. The peak intensity continued to decrease gradually for 12 hours. More interestingly during the grafting process, the bottom part of the peak broadened continuously while the top part remained sharp, suggesting the existence of two possible types of PEG chains in solution. The presence of two types of PEG chains was further confirmed by diffusion-ordered spectroscopy (DOSY) as two peaks appeared at $3.70 \mathrm{ppm}$ (Fig. 1c and S2 $\dagger$ ). The diffusion coefficients of these two peaks were $8.07 \times 10^{-11}$ (free PEG) and $1.39 \times 10^{-11} \mathrm{~m}^{2} \mathrm{~s}^{-1}$ (grafted PEG); peak assignments were made after comparing the data with the diffusion coefficient of pure GNPs $\left(1.91 \times 10^{-11} \mathrm{~m}^{2} \mathrm{~s}^{-1}\right)$, determined by dynamic light scattering measurements (Fig. S3 $\dagger$ ). On the basis of these results, the NMR peak at 3.70 ppm was a superposition of signals from free PEG molecules and PEG grafted on the NP surface.

It is known that the attachment to NP surfaces causes the NMR signal of a ligand molecule to broaden and sometimes disappear, because the particle-bound ligand has faster transverse relaxation times $\left(T_{2}\right)$ leading to line shape broadening according to the following equation: ${ }^{33}$

$$
v_{\mathrm{HWHM}}=\frac{1}{\pi T_{2}},
$$

where $v_{\text {HWHM }}$ is the half width at half maximum (HWHM) of the NMR signal. ${ }^{31,34-36}$ Once grafted onto the surface of GNPs, the PEG molecules lost most of their rotational freedom as the GNPs possessed a much larger hydrodynamic radius than that of free, dispersed PEG molecules. As a result, the grafted PEG molecules experienced slower molecular tumbling and correlation time in solutions, leading to a decrease of $T_{2}$ and an increase of $v_{\text {HWHм }} \cdot{ }^{37-40}$ As the peak of the grafted PEG became indistinguishable with the baseline of NMR spectra, it is not possible to quantify the grafted PEG concentration by integrating the area under the peak (Fig. S4†). On the other hand, the integral of the peak of free PEG molecules should remain proportional to the number of protons on them, if one can distinguish the peak of free PEG from that of grafted PEG.

Once the concentration of free PEG was determined, the $\sigma$ of PEG on the GNPs can be calculated as:

$$
\sigma=\frac{[\mathrm{PEG}]_{\mathrm{grafted}}}{4 \pi R^{2}[\mathrm{GNP}]}=\frac{[\mathrm{PEG}]_{\mathrm{tot}}-[\mathrm{PEG}]_{\mathrm{free}}}{4 \pi R^{2}[\mathrm{GNP}]},
$$

where $R$ is the average radius of the GNPs, [GNP] is the molar concentration of GNPs, and $[\mathrm{PEG}]_{\text {tot }},[\mathrm{PEG}]_{\text {grafted }}$, and $[\mathrm{PEG}]_{\text {free }}$ is the molar concentration of the total, grafted, and free PEG in solution, respectively. As mentioned above, $[\mathrm{PEG}]_{\text {free }}$ can be obtained from the integral of the NMR peak of free PEG molecules. We then developed an algorithm to distinguish the NMR peak of free PEG from that of grafted PEG in order to calculate the grafting density of PEG on the GNPs. Specifically, the line shape of an NMR peak is determined by the free induction decay (FID) of the observable NMR signal generated by nonequilibrium nuclear-spin magnetization precession about the magnetic field. The Fourier transform of this two-sided decaying exponential function is a Lorentzian function, which is represented as $\varphi\left(\delta ; \delta_{0}, \gamma\right)$,

$$
\varphi\left(\delta ; \delta_{0}, \gamma\right)=\frac{1}{\pi \gamma}\left[\frac{\gamma^{2}}{\left(\delta-\delta_{0}\right)^{2}+\gamma^{2}}\right],
$$

where $\delta_{0}$ is the location parameter (the chemical shift of the NMR peak in ppm) and $\gamma$ is the scale parameter which specifies the $v_{\text {HWHM }}$ of the peak. In the present work, free PEG in $\mathrm{D}_{2} \mathrm{O}$ has 

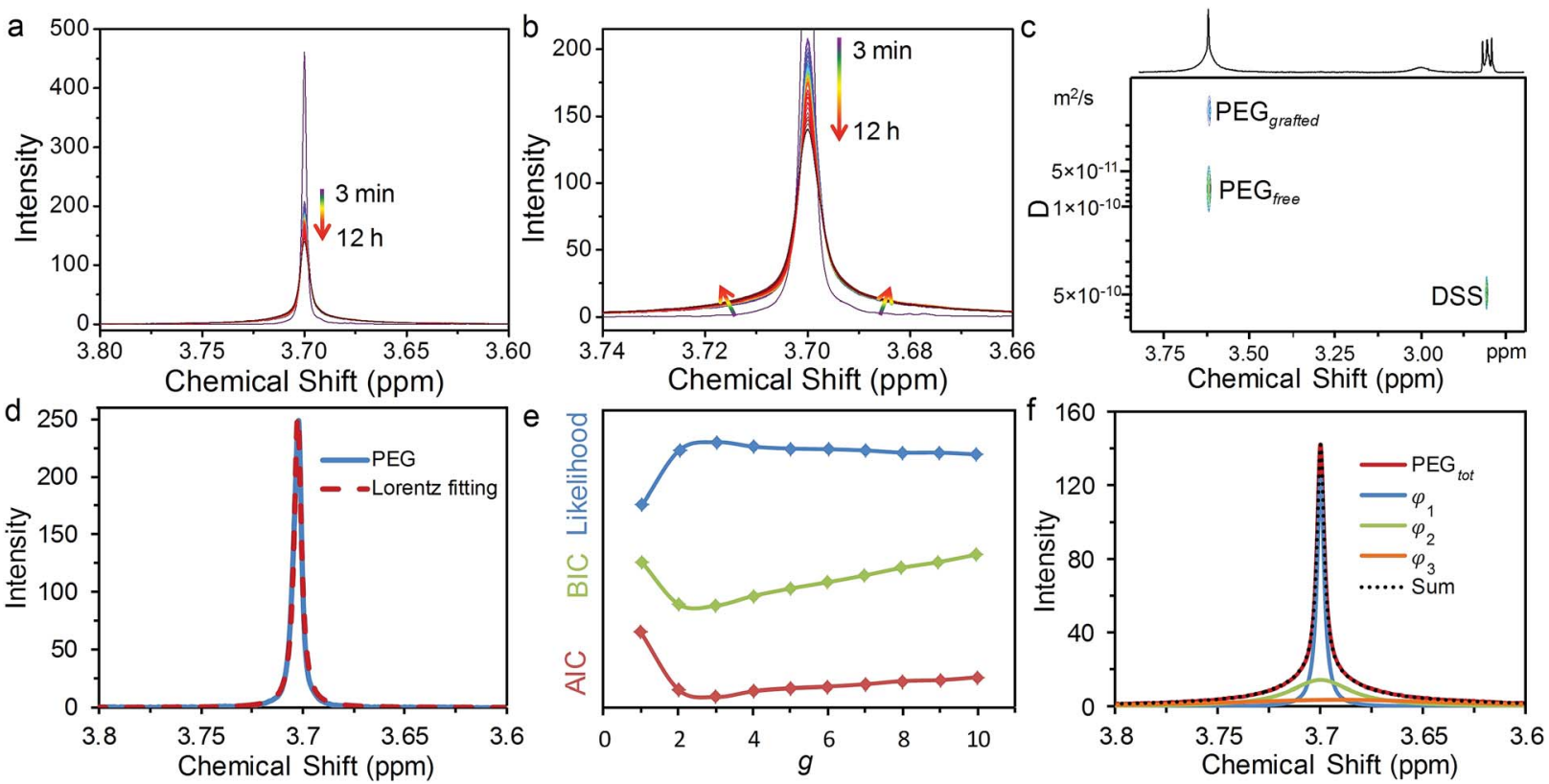

Fig. 1 Temporal evolution of ${ }^{1} \mathrm{H}$ NMR spectra ( $a$ and $b$ ) to monitor the grafting process of HS-PEG onto the surface of GNPs in $\mathrm{D}_{2} \mathrm{O}$. The evolution of the colour from violet to red indicated an increase in grafting-to time. NMR spectra were obtained by following the grafting process up to $12 \mathrm{~h}$ with a variable time interval of 1 (3-5 min), 5 (5-120 min), or $30 \mathrm{~min}$ (120-720 min). (c) DOSY spectrum of the PEG signal after graftingto the surface of GNPs. (d) Lorentz fitting for ${ }^{1} \mathrm{H}$ NMR spectra of pure PEG molecules. (e) Evolution of log likelihood (blue), BIC (green) and AIC (red) with the number of components, $g$. (f) Multi-Lorentzian-splitting to isolate free PEG from the mixtures with grafted PEG using three evaluation criterions.

only one singlet at $3.70 \mathrm{ppm}$, whose line shape can be well-fitted by a Lorentzian function defined as $\varphi\left(\delta ; \delta_{0}, \gamma_{1}\right)$ with $\delta_{0}$ of $3.70, \gamma_{1}$ of 0.00215 , and a coefficient of determination $\left(R^{2}\right)$ of 0.994 (Fig. 1d). In comparison, the grafted PEG molecules generate one or more relatively boarder NMR peaks because of a distribution of the grafted PEG chains on GNPs and a range of distances between the protons of grafted PEG chains and the electrons on the GNP surface. ${ }^{31}$ The Lorentzian function for the line shape of each proton of the grafted PEG can be represented as $\varphi\left(\delta ; \delta_{i}, \gamma_{i}\right)$. Compared to the function for free PEG, $\delta_{i}$ is equal to $\delta_{1}$ as the peak position does not change but $\gamma_{i}$ is greater than $\gamma_{1}$ as the line width of grafted PEG is larger than that of free PEG. Therefore, NMR peaks of a complex consisting of $g$ components can be represented in a linear form of Lorentzian functions:

$$
f\left(\delta ; \phi_{g}\right)=\sum_{i=1}^{g} A_{i} \varphi\left(\delta ; \delta_{i}, \gamma_{i}\right),
$$

where $\phi_{g}$ is a vector for the parameters of each Lorentzian function. $A_{i}, \delta_{i}$, and $\gamma_{i}$ represent peak area (proportional to the PEG concentration), chemical shift, and $v_{\mathrm{HWHM}}$ of the corresponding component.

To isolate the Lorentzian function contribution from the free PEG, $\phi_{g}$ and the most probable number of components $(g)$ need to be identified. For this purpose, we developed a multiLorentzian-splitting algorithm to estimate $g$ and the corresponding Lorentzian functions. Firstly, Monte Carlo (MC) simulations with sufficient steps $(>10000)$ were performed to generate samples from the original NMR data with chemical shifts in the range of 3.4 to $4.0 \mathrm{ppm}$. Afterwards, $\phi_{g}$ was fitted using the least-square algorithm for $g$ ranging from one to ten components. Next, the fitting results were evaluated to find the most probable $g$ using the criteria of the lowest Akaike's information criterion (AIC), the lowest Bayesian information criterion (BIC), and the maximum likelihood estimation. ${ }^{41-43}$ Noted that these three criteria may give different values of $g$ because using AIC/BIC may give a value of $g$ different from that using the maximum likelihood estimation. AIC and BIC are theoretically equivalent; ${ }^{43}$ therefore they are normally satisfied at the same time for a specific value of $g$. As the values from AIC and BIC heavily rely on specific dataset, the criterion with max difference between the values from BIC and AIC are always chosen for analysis in practical application. The maximum likelihood estimation is auxiliary to choose $g$. Its value is relatively small and easily affected by data fluctuations; therefore the maximum likelihood estimation is possible to give inconsistent results with AIC/BIC criteria. However, as the fluctuations of the NMR data are very small, these three criteria can give the same value of $g$.

Using the NMR spectrum at a grafting time of 720 minutes as an example, the AIC, BIC and maximum likelihood estimation all gave a $g$ of 3 as the best fit (Fig. 1e). This result indicates that three peaks should be used for the multiple-Lorentzian splitting of the superposed NMR peak from both free and grafted PEG, including one sharp and two relatively broad peaks (Fig. 1f). The $\gamma$ for the sharp peak is equal to 0.00210 , which is very close to that of the pure PEG molecules $\left(\gamma_{1}=0.00215\right.$ in Fig. $\left.1 d\right)$, suggesting that the sharp peak should be assigned to free PEG. The 
other two peaks with higher $\gamma$ values $(0.0182$ and 0.0680$)$ are assigned to grafted PEG. After comparing the integral of free PEG with the standard curve of pure PEG, the concentrations of free PEG, grafted PEG and corresponding $\sigma$ can be calculated.

\section{Grafting process of HS-PEG onto gold nanoparticles}

We used this in situ NMR spectroscopy to analyse the grafting process of PEG onto the surface of GNPs (Fig. 2). Fig. 2a shows the temporal evolution of the ${ }^{1} \mathrm{H}$ NMR spectra of free PEG, isolated from the NMR spectra in the grafting process (Fig. 1) via the multi-Lorentzian-splitting algorithm. Based on the integral of free PEG in ${ }^{1} \mathrm{H}$ NMR spectra, concentrations of free PEG at any time during the grafting process can be calculated. Fig. $2 \mathrm{~b}$ shows the evolution of $\sigma$ with grafting time. The $\sigma$ was determined to be 1.34 chains per $\mathrm{nm}^{2}$ at $t=3$ minutes, indicating the grafting process was fairly quick due to the strong affinity between gold and sulphur atoms. ${ }^{44}$ For longer grafting times of several minutes to about 180 minutes, $\sigma$ gradually increased to 1.53 chains per $\mathrm{nm}^{2}$. At the end of the grafting process $(720$ $\min ), \sigma$ eventually reached a maximum of 1.55 chains per $\mathrm{nm}^{2}$ for full coverage.

We also evaluated the accuracy and precision of the multiLorentzian splitting method. The accuracy of this approach was verified by a post-centrifugation strategy in which the GNPs with grafted PEG was removed from the solution by multiple centrifugation to obtain free PEG supernatant for ${ }^{1} \mathrm{H}$ NMR quantification (Fig. S5a $\dagger$ ). Using GNPs (13 nm, capped by citrate) grafted with HS-PEG $\left(M_{\mathrm{w}}\right.$ of $\left.5.0 \mathrm{~kg} \mathrm{~mol}^{-1}\right)$ as an example, the NMR peak of free PEG from the multi-Lorentzian-splitting algorithm was highly coincided with that from the post-
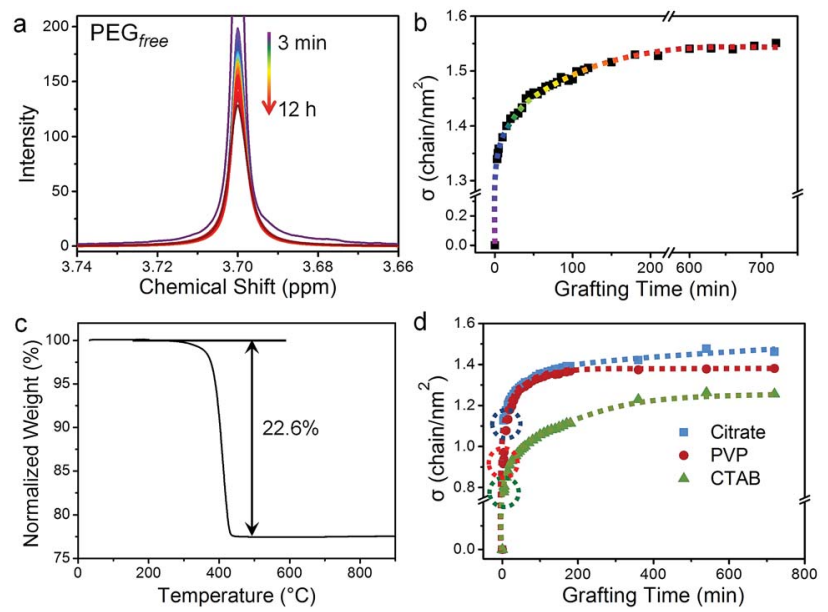

Fig. 2 Grafting process for HS-PEG onto the surface of GNPs analysed by the multi-Lorentzian-splitting algorithm. Temporal evolution of ${ }^{1} \mathrm{H}$ NMR spectra of (a) free PEG and (b) $\sigma$ in the grafting-to process of HSPEG onto the surface of citrate-capped GNPs. The colour of the lines corresponds to the same time points as Fig. 1. (c) Thermal curve from thermal gravimetric analysis (TGA) of HS-PEG grafted GNPs. (d) Temporal evolution of $\sigma$ for HS-PEG grafted onto the surface of GNPs capped by citrate (blue squares), PVP (red circles), and CTAB (green triangles). Dotted circles on the curves in (d) highlight $\sigma$ at the grafting time of $3 \mathrm{~min}$. centrifugation strategy, and the calculated $\sigma$ at saturation (1.55 chains per $\mathrm{nm}^{2}$ ) was almost identical with that from the post-centrifugation strategy ( 1.53 chains per $\mathrm{nm}^{2}$ ) (Fig. S5b and Table S1 $\dagger$ ). The accuracy of the multi-Lorentzian-splitting algorithm can also be confirmed by comparison with other quantification methods such as TGA, TOC and analytical ultracentrifugation (AUC). The value of $\sigma\left(1.55\right.$ chains per $\left.\mathrm{nm}^{2}\right)$ determined from the multi-Lorentzian-splitting method is very close to that from TGA (1.44 chains per $\mathrm{nm}^{2}$, Fig. $2 \mathrm{c}$ ). It should be noted $\sigma$ determined from TGA could be underestimated as PEG can potentially detach from GNPs during repeated centrifugations. This $\sigma$ for $13 \mathrm{~nm}$ GNPs determined from NMR spectroscopy is also in reasonable agreement with $\sigma$ reported for the same HS-PEG on $11 \mathrm{~nm}$ GNPs (measured by TOC and AUC; 1.77 chains per $\mathrm{nm}^{2}$ ), where the smaller GNPs allow for denser packing and a higher $\sigma .^{29} \mathrm{Next}$, the precision of the multi-Lorentzian-splitting method was evaluated via repeated measurements under identical experimental and analytical conditions, i.e. three replicas for quantifying $\sigma$ of GNPs with different sizes and PEG with different molecular weights (Fig. 3). The majority of the standard deviation values obtained with this method were less than $5 \%$, and the maximum standard deviation was no more than $8 \%$. These results prove that the multi-Lorentzian-splitting method is highly accurate with excellent reproducibility.

\section{Effect of capping ligands}

The capping ligands on the surface of GNPs play a critical role during ligand exchange with the HS-PEG molecules. To study the effect of ligands on the grafting process, GNPs capped with three different kinds of ligands, i.e. citrate, cetyltrimethylammonium bromide (CTAB), and polyvinylpyrrolidone (PVP), were reacted with HS-PEG under identical grafting conditions. $13 \mathrm{~nm}$ citrate-coated GNPs were obtained referred to the standard Turkevich method. ${ }^{45}$ The other two types of GNPs of the same size were prepared via ligand exchange of CTAB or PVP with the citrate-covered GNPs, made possible as CTAB and PVP possess stronger binding affinity for GNPs than citrate. ${ }^{26}$ The successful ligand exchanges were confirmed by UV and zeta-potential measurements (Fig. S6†).

The grafting processes of HS-PEG onto citrate-, CTAB-, and PVP-covered GNPs were monitored by ${ }^{1} \mathrm{H}$ NMR spectroscopy and analysed with the multi-Lorentzian-splitting algorithm (Fig. S7 $\dagger$ ). Fig. $2 d$ shows the temporal evolution of $\sigma$ for HS-PEG $\left(M_{\mathrm{w}}\right.$ of $\left.5.0 \mathrm{~kg} \mathrm{~mol}{ }^{-1}\right)$ grafted onto the surfaces of GNP@citrate, GNP@PVP and GNP@CTAB, respectively. At the grafting time of $3 \mathrm{~min}, \sigma$ decreased in the order of GNP@citrate (1.13 chains per $\left.\mathrm{nm}^{2}\right)>$ GNP@PVP (0.92 chains per $\left.\mathrm{nm}^{2}\right)>$ GNP@CTAB (0.78 chains per $\mathrm{nm}^{2}$ ), indicating that the grafting rate was inversely correlated to the binding affinity of the original capping ligand on the surface of GNPs (CTAB > PVP > citrate).$^{26}$ As grafting continued, $\sigma$ of these GNPs capped by different ligands exhibited a similar trend such that $\sigma$ increased progressively from several minutes to about $180 \mathrm{~min}$, and then reached a full coverage state. The maximum $\sigma$ attained decreased in the order 
of GNP@citrate (1.46 chains per nm $\left.{ }^{2}\right)>$ GNP@PVP (1.38 chains per $\mathrm{nm}^{2}$ ) > GNP@CTAB (1.26 chains per $\mathrm{nm}^{2}$ ), which was the opposite of the binding affinity trend observed for the original capping ligands, revealing that the maximum $\sigma$ was also inversely correlated to the binding affinity of the capping ligands on GNPs.

\section{Effect of nanoparticle size}

It is well known that the size of NPs, i.e. the curvature of NP surface, plays an important role on grafting density. However, studies on the effect of NP sizes on grafting rate are very rare due to a lack of practical characterization methods. ${ }^{31}$ To further demonstrate the power of our method, we exploited this multiLorentzian-splitting algorithm to evaluate the effect of GNP sizes on the grafting process. For this purpose, monodisperse CTAB-capped GNPs with average diameters ranging from 8 to $42 \mathrm{~nm}$ and standard deviations of less than $10 \%$ were conjugated with HS-PEG in $\mathrm{D}_{2} \mathrm{O}$ solutions $\left(M_{\mathrm{w}}\right.$ of $5.0 \mathrm{~kg} \mathrm{~mol}^{-1}$, [PEG] of $0.60 \mathrm{mg} \mathrm{mL}^{-1}$ ) (Fig. S8 and S9†). For each GNP size, the initial [GNP] used was inversely proportional to the surface area of NPs (the square of the diameter) to keep the total surface area of GNPs the same. The multi-Lorentzian-splitting algorithm was then used to calculate $\sigma$ for each NP size. Fig. 3a shows the temporal evolution of $\sigma$ for HS-PEG on GNPs with average diameters $(d)$ of 8.5, 16.2, 28.0, and $41.9 \mathrm{~nm}$. At the grafting time $(t)$ of $3 \mathrm{~min}, \sigma$ decreased with increasing size of GNPs, calculated as 1.08, 0.82, 0.69 and 0.48 chains per $\mathrm{nm}^{2}$ for $d_{\mathrm{GNP}}$ of 8.5, 16.2, 28.0 , and $41.9 \mathrm{~nm}$, respectively. Note that $[\mathrm{GNP}]_{d=8.5}$ was almost 25 times greater than $[\mathrm{GNP}]_{d=41.9}$, meaning that the relative ratio of HS-PEG to GNPs for smaller GNPs was much less than that of larger GNPs. The higher $\sigma$ observed for smaller GNPs indicates that the grafting rate is strongly dependent on the
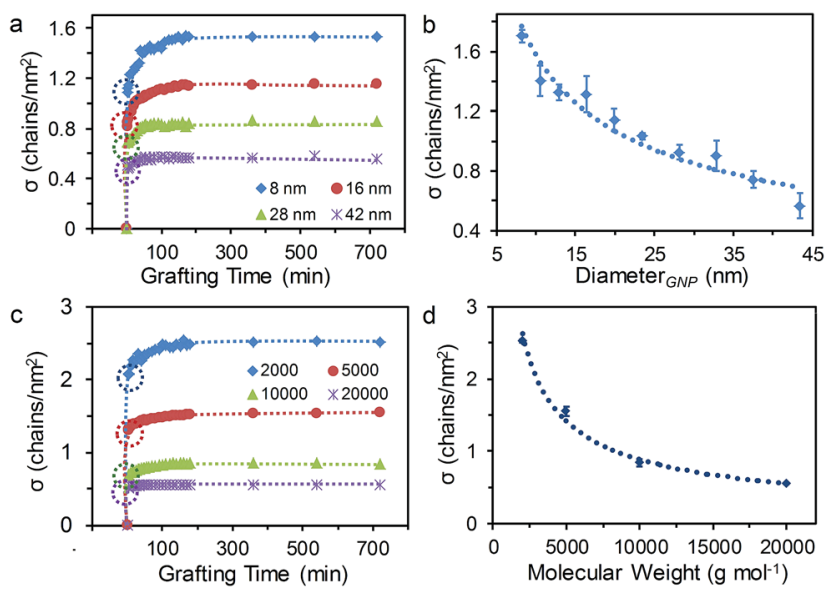

Fig. 3 ( $(\mathrm{a}$ and $\mathrm{c}$ ) Temporal evolution of $\sigma$ for the grafting process of HSPEG on GNPs and plot of $\sigma$ at full coverage against (b) the diameter of NPs and (d) HS-PEG molecular weight. The polymer molecular weight, original capping ligand and NP size were as follows: (a and b) $5.0 \mathrm{~kg}$ $\mathrm{mol}^{-1} \mathrm{HS}$-PEG grafted to CTAB-capped NPs in various sizes, and (c and d) various molecular weights of HS-PEG grafted to $13 \mathrm{~nm}$ citratecapped NPs. Dotted circles in (a) and (c) highlight $\sigma$ at the grafting time of $3 \mathrm{~min}$. The error bars in (b) represent standard deviation from three replicas each measurement. diffusion rate of GNPs, which is inversely proportional to their diameters (Stokes-Einstein equation). ${ }^{46}$ The grafting time to achieve a fully covered GNP surface with HS-PEG decreased from 180 to 50 min with increasing GNP sizes, primarily a result of the much denser surface coverage (i.e. higher $\sigma$ at saturation) of smaller GNPs compared to larger ones. As expected, the plot $\sigma$ at saturation against the diameter of GNPs revealed that $\sigma$ decreased from 1.70 to 0.56 chains per $\mathrm{nm}^{2}$ while $d$ increased from 8.5 to $41.9 \mathrm{~nm}$ (Fig. 3b). This indicates that the grafting efficiency of PEG onto the surface of GNPs was reduced with increasing curvature of GNPs. This result is qualitatively consistent with previous studies of other ligands bound to the surface of nanocrystals. ${ }^{47-49}$

\section{Effect of the molecular weight of HS-PEG}

We also used this method to study the effect of HS-PEG molecular weights on the grafting process. Fig. $3 \mathrm{c}$ and $\mathrm{S} 10^{\dagger} \dagger$ show the temporal evolution of $\sigma$ and the NMR signal, respectively, for a series of HS-PEG with increasing molecular weight $\left(M_{\mathrm{w}}\right.$ of $2.0,5.0,10.0$ and $20.0 \mathrm{~kg} \mathrm{~mol}^{-1}$, [PEG] of $0.50 \mathrm{mg} \mathrm{mL}^{-1}$ ) grafting onto $13 \mathrm{~nm}$ citrate-capped GNPs. These experiments revealed that the grafting process was dramatically affected by the HS-PEG chain length. Similar to the trend of $\sigma$ for decreasing GNP sizes, the grafting rate increased with decreasing HS-PEG chain length. This result suggests the grafting process is dependent on the diffusivity of free HS-PEG molecules, which decreases exponentially in water as the chain length is increased. ${ }^{49}$ This observation is also consistent with our previous study on the grafting process of HS-PEG on a flat $\mathrm{Au}$ surface. ${ }^{50}$ Plotting the $\sigma$ at saturation (i.e. equilibrium) against the molecular weights demonstrated that $\sigma$ decreased in the order of 2.53, 1.55, 0.84, 0.55 chains per $\mathrm{nm}^{2}$ for $M_{\mathrm{w}}$ of 2.0, $5.0,10.0$ and $20.0 \mathrm{~kg} \mathrm{~mol}^{-1}$, respectively. The data confirmed that $\sigma$ at saturation decreased with increasing molecular weight (Fig. 3d), which is consistent with previous studies of $\sigma$ for various polymer molecular weights. ${ }^{29,51}$

\section{Limitations of the multi-Lorentzian-splitting algorithm}

The limitations of our method are mainly determined by the following aspects: (1) the size of gold nanoparticles, (2) the molecular weight of PEG molecules, and (3) the fraction of free PEG among all PEG molecules in reaction solutions. For the multi-Lorentzian-splitting algorithm, the separation of the NMR signals of free PEG from the grafted ones is dependent on their peak-width difference, which is caused by the slow diffusion of PEG on the nanoparticle surface. Therefore, the multiLorentzian-splitting algorithm is not suitable for the scenario when the diffusion of grafted PEG or part of it is similar to that of free PEG, that is when the size of nanoparticles is too small, or the molecular weight of PEG is too large.

For the limitation of GNP size, we determined the lower limit of nanoparticle size by comparing the grafting density obtained by the multi-Lorentzian-splitting algorithm and postcentrifugation methods (Fig. S11†). The latter is highly accurate for the quantification of final grafting densities, but not capable for monitoring the grafting process. Fig. S11 $\uparrow$ reveals 
that the lower limit of nanoparticle size of the multi-Lorentziansplitting algorithm increases with the molecular weight of PEG molecules. For example, the lower limit of nanoparticle size for PEG-5K is $c a .10 \mathrm{~nm}$, while that for PEG-20K is $c a .23 \mathrm{~nm}$. In principle, there is no upper limit of nanoparticle size for the multi-Lorentzian-splitting algorithm, as the difference of diffusion between free PEG and grafted one can only increase with the size of nanoparticles. It should be noticed that when the size of nanoparticles is too large, the NMR signal of grafted PEG will become too broad to distinguish from the spectral baseline. Therefore, one can directly quantify the amount of free PEG from the NMR peak of PEG in reaction solutions.

For biomedical applications of PEGylated Au nanoparticles, the molecular weight of PEG is generally in the range of 2.0 to 20 $\mathrm{kg} \mathrm{mol}{ }^{-1}$. We found the multi-Lorentzian-splitting is suitable for the PEG in this range. The grafting density of PEG with shorter chain lengths (i.e. molecular weight less than $2.0 \mathrm{~kg}$ $\mathrm{mol}^{-1}$ ) could be determined by the previous methods developed for small molecular ligands. For PEG with longer chain lengths (i.e. molecular weight higher than $20 \mathrm{~kg} \mathrm{~mol}^{-1}$ ), the multiLorentzian-splitting algorithm can be used to determine the grafting density of PEG on NPs with sizes above the lower limit for each molecular weight.

In addition, the multi-Lorentzian-splitting algorithm method is more appropriate for the scenario when the NMR signal of free PEG is not significantly larger than that of grafted ones, that is the fraction of free PEG among all PEG is relatively small. In a controlled experiment, we continuously added free PEG-5K into a solution of PEGlyated GNPs (size of $13 \mathrm{~nm}$ ) with saturate and known grafting density of the same PEG (1.53 chains per $\mathrm{nm}^{2}$ ). The ${ }^{1} \mathrm{H}$ NMR signals for the solution with different free/total PEG ratios were interpreted by the multiLorentzian-splitting algorithm to obtain the calculated grafting density, which were compared to the known value as shown in Fig. S12. $\dagger$ The comparison result reveals that once the fraction of free PEG was beyond $60 \%$, the calculated grafting density determined by the multi-Lorentzian-splitting algorithm became inconsistent with the actual known value. In the scenario when the fraction of free PEG is much larger than that of the grafted ones, the grafting density will become saturated and can be easily determined by the post-centrifugation method.

\section{Quantification of PEG grafting-to $\mathrm{SiO}_{2}$ encapsulated gold nanorods}

The grafting-to process of PEG on NP surfaces is a thermodynamically-driven adsorption/desorption process, and should be affected by the activity of the adsorption reaction of ligands with the NP surfaces. Despite the reaction of thiol with gold, other adsorptions through supramolecular interactions or covalent reactions can co-exist and the corresponding grafting densities need to be quantified. In order to verify the universal applicability of our multi-Lorentzian-splitting algorithm to other types of adsorption reactions, we selected the PEGylation of $\mathrm{SiO}_{2}$ encapsulated gold nanorods (GNR@SiO${ }_{2}$ ) as an example because of their potential biomedical applications such as photothermal therapy, ${ }^{52}$ photoacoustic imaging, ${ }^{53}$ biosensing, ${ }^{54}$ and drug delivery. ${ }^{55}$ Three typical adsorption reactions were used to graft PEG chains $\left(M_{\mathrm{w}}\right.$ of $\left.5.0 \mathrm{~kg} \mathrm{~mol}{ }^{-1}\right)$ onto $\mathrm{GNR@SiO}$ surfaces (Fig. 4a, b and $\mathrm{S} 13 \dagger$ ). The first reacted aminefunctionalized GNR@SiO $\left(\mathrm{GNR@SiO}-\mathrm{NH}_{2}\right)$ with $\alpha$-methoxy$\omega$-carboxyl poly(ethylene glycol) (PEG-COOH) via electronic interactions (Route I). The second employed GNR@SiO ${ }_{2}-\mathrm{NH}_{2}$ to interact with $\alpha$-methoxy- $\omega$ - $N$-hydroxysuccinimide-polyethylene glycol (PEG-NHS) by the formation of active esters (Route II). The third involved a hydrolysis reaction of 2-[methox$\mathrm{y}$ (polyethyleneoxy)propyl]trimethoxysilane (PEG-silane) on $\mathrm{SiO}_{2}$ shells covered GNRs under acidic conditions (Route III). ${ }^{\mathbf{5 6}}$

In order to quantify the grafting density for each route, the multi-Lorentzian-splitting algorithm was applied to isolate the free PEG signal from the mixture containing grafted PEG after the grafting-to process reached equilibrium. Fig. 4c shows the NMR spectra of free PEG calculated via the multi-Lorentziansplitting method. The grafting density for each route was determined by comparing the integral of the free PEG signal with the standard curve of pure PEG (Fig. 4d). Route III yielded the highest grafting efficiency with $\sigma$ up to 0.78 chain per $\mathrm{nm}^{2}$, which was 43 and 15 times larger than that of routes I $(0.018$ chain per $\left.\mathrm{nm}^{2}\right)$ and II ( 0.051 chain per $\left.\mathrm{nm}^{2}\right)$, respectively; this revealed that the hydrolysis of PEG-silane is a much more effective way to graft PEG chains onto $\mathrm{SiO}_{2}$ shells covered GNRs. It should be noted that during the hydrolysis reaction, there is no by-products, i.e. PEG micelles, observed by the ${ }^{1} \mathrm{H}$ NMR and dynamic light scattering (DLS) studies (Fig. S14, Table S2 and the discussion in ESI $\dagger$ ). Additionally, $\sigma$ achieved through Route I and II were similar to $\sigma$ of HS-PEG on CTAB-covered GNRs, which was determined as 0.034 chain per $\mathrm{nm}^{2}$ by the same multi-Lorentzian-splitting algorithm (Fig. S15†). Taken together, these results indicate that the multi-Lorentziansplitting algorithm is well-suited to quantify the coverage from PEG ligands in various adsorption reactions with NP surfaces and is not limited by the NP shapes.

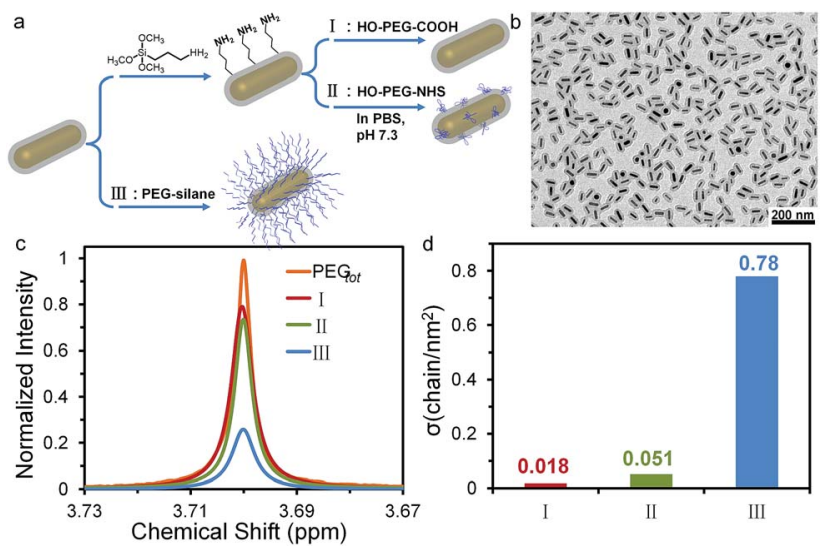

Fig. 4 (a) Three reaction routes for PEGylation of $\mathrm{GNR}_{\mathrm{CSiO}}$. (b) TEM image of $\mathrm{GNRASiO}_{2}$. (c) ${ }^{1} \mathrm{H}$ NMR spectra of free PEG calculated via the multi-Lorentzian-splitting algorithm after the three grafting-to processes reached equilibrium, along with the initial spectrum before the grafting process. (d) Corresponding $\sigma$ calculated by the multiLorentzian-splitting algorithm for the three routes. 


\section{Conclusions}

In summary, we report a facile and non-sacrificial ${ }^{1} \mathrm{H}$ NMR analytical approach to quantitatively characterize PEG grafting density on GNPs. In order to distinguish the NMR signal of free PEG from that of grafted molecules, a multi-Lorentziansplitting algorithm is proposed and allows monitoring of the grafting process in situ. Using this method, the grafting rates and grafting densities can be efficiently studied for GNPs with different dimensions, PEG with different molecular weights, and different types of the original capping ligand on GNPs. This method is not limited by the types of terminal functional group of PEG, the surface chemistry of nanoparticles, or the composition of nanoparticles, and can be applied to other polymer ligands capping different types of nanoparticles. More importantly, our methods provide a set of critical and standard guide for characterization of the PEG grafting density on nanoparticles (the most important parameter in biological interactions) for in vivo biological and biomedicine studies. ${ }^{57,58}$

\section{Conflicts of interest}

There are no conflicts to declare.

\section{Acknowledgements}

K. L. and Z.-Y. L. gratefully acknowledge financial support from the National Natural Science Foundation of China (21534004). K. L. thanks the National Natural Science Foundation of China (21474040, 21674042) and China's Thousand Talent Plan for financial support.

\section{Notes and references}

1 C. J. Murphy, A. M. Gole, J. W. Stone, P. N. Sisco, A. M. Alkilany, E. C. Goldsmith and S. C. Baxter, Acc. Chem. Res., 2008, 41, 1721.

2 N. L. Rosi and C. A. Mirkin, Chem. Rev., 2005, 105, 1547.

3 Z. Zhang, J. Wang and C. Chen, Adv. Mater., 2013, 25, 3869.

4 C. Tu, S. Das, A. B. Baker, J. Zoldan and L. J. Suggs, ACS Nano, 2015, 9, 3436.

5 S. Wilhelm, A. J. Tavares, Q. Dai, S. Ohta, J. Audet, H. F. Dvorak and W. C. W. Chan, Nat. Rev. Mater., 2016, 1, 16014.

6 J. Lazarovits, Y. Y. Chen, E. A. Sykes and W. C. W. Chan, Chem. Commun., 2015, 51, 2756.

7 J. W. Nichols and Y. H. Bae, Nano Today, 2012, 7, 606.

8 E. Blanco, H. Shen and M. Ferrari, Nat. Biotechnol., 2015, 33, 941.

9 I. Lynch and K. A. Dawson, Nano Today, 2008, 3, 40.

10 M. Lundqvist, J. Stigler, G. Elia, I. Lynch, T. Cedervall and K. A. Dawson, Proc. Natl. Acad. Sci. U. S. A., 2008, 105, 14265.

11 C. D. Walkey and W. C. W. Chan, Chem. Soc. Rev., 2012, 41, 2780.

12 A. E. Nel, L. Mädler, D. Velegol, T. Xia, E. M. V. Hoek, P. Somasundaran, F. Klaessig, V. Castranova and M. Thompson, Nat. Mater., 2009, 8, 543.
13 S. M. Moghimi, A. C. Hunter and J. C. Murray, Pharmacol. Rev., 2001, 53, 283.

14 X. Yang, M. Yang, B. Pang, M. Vara and Y. Xia, Chem. Rev., 2015, 115, 10410.

15 J. S. Suk, Q. Xu, N. Kim, J. Hanes and L. M. Ensign, Adv. Drug Delivery Rev., 2016, 99, 28.

16 V. C. F. Mosqueira, P. Legrand, J.-L. Morgat, M. Vert, E. Mysiakine, R. Gref, J.-P. Devissaguet and G. Barratt, Pharm. Res., 2001, 18, 1411.

17 J. V. Jokerst, T. Lobovkina, R. N. Zare and S. S. Gambhir, Nanomedicine, 2011, 6, 715.

18 R. Gref, M. Lück, P. Quellec, M. Marchand, E. Dellacherie, S. Harnisch, T. Blunk and R. H. Müller, Colloids Surf., B, 2000, 18, 301.

19 L. D. Unsworth, H. Sheardown and J. L. Brash, Langmuir, 2008, 24, 1924.

20 Q. Yang, S. W. Jones, C. L. Parker, W. C. Zamboni, J. E. Bear and S. K. Lai, Mol. Pharmaceutics, 2014, 11, 1250.

21 C. H. J. Choi, C. A. Alabi, P. Webster and M. E. Davis, Proc. Natl. Acad. Sci. U. S. A., 2010, 107, 1235.

22 M. Maccarini, G. Briganti, S. Rucareanu, X.-D. Lui, R. Sinibaldi, M. Sztucki and R. B. Lennox, J. Phys. Chem. C, 2010, 114, 6937.

23 E. Mansfield, A. Kar, T. P. Quinn and S. A. Hooker, Anal. Chem., 2010, 82, 9977.

24 M. K. Corbierre, N. S. Cameron, M. Sutton, K. Laaziri and R. B. Lennox, Langmuir, 2005, 21, 6063.

25 H. Zhang, W. Wang, S. Mallapragada, A. Travesset and D. Vaknin, Nanoscale, 2017, 9, 164.

26 X. Xia, M. Yang, Y. Wang, Y. Zheng, Q. Li, J. Chen and Y. Xia, ACS Nano, 2012, 6, 512.

27 C. S. Levin, S. W. Bishnoi, N. K. Grady and N. J. Halas, Anal. Chem., 2006, 78, 3277.

28 H. Hinterwirth, S. Kappel, T. Waitz, T. Prohaska, W. Lindner and M. Lämmerhofer, ACS Nano, 2013, 7, 1129.

29 D. N. Benoit, H. Zhu, M. H. Lilierose, R. A. Verm, N. Ali, A. N. Morrison, J. D. Fortner, C. Avendano and V. L. Colvin, Anal. Chem., 2012, 84, 9238.

30 V. B. Damodaran, C. J. Fee, T. Ruckh and K. C. Popat, Langmuir, 2010, 26, 7299.

31 A. M. Smith, L. E. Marbella, K. A. Johnston, M. J. Hartmann, S. E. Crawford, L. M. Kozycz, D. S. Seferos and J. E. Millstone, Anal. Chem., 2015, 87, 2771.

32 K. A. Johnston, A. M. Smith, L. E. Marbella and J. E. Millstone, Langmuir, 2016, 32, 3820.

33 M. H. Levitt, Spin Dynamics: Basics of Nuclear Magnetic Resonance, John Wiley \& Sons Ltd., West Sussex, England, 2nd edn, 2008.

34 M. J. Hostetler, J. E. Wingate, C.-J. Zhong, J. E. Harris, R. W. Vachet, M. R. Clark, J. D. Londono, S. J. Green, J. J. Stokes, G. D. Wignall, G. L. Glish, M. D. Porter, N. D. Evans and R. W. Murray, Langmuir, 1998, 14, 17.

35 E. Tavasoli, Y. Guo, P. Kunal, J. Grajeda, A. Gerber and J. Vela, Chem. Mater., 2012, 24, 4231.

36 C.-C. Lin, Y. Guo and J. Vela, ACS Catal., 2015, 5, 1037. 
37 O. Kohlmann, W. E. Steinmetz, X.-A. Mao, W. P. Wuelfing, A. C. Templeton, R. W. Murray and C. S. Johnson, J. Phys. Chem. B, 2001, 105, 8801.

38 L. E. Marbella and J. E. Millstone, Chem. Mater., 2015, 27, 2721.

39 Z. Hens and J. C. Martins, Chem. Mater., 2013, 25, 1211.

40 J. D. Roo, N. Yazdani, E. Drijvers, A. Lauria, J. Maes, J. S. Owen, I. V. Driessche, M. Niederberger, V. Wood, J. C. Martins, I. Infante and Z. Hens, Chem. Mater., 2018, 30, 5485 .

41 D. F. Findley, Ann. Inst. Stat. Math., 1991, 43, 505.

42 H. Akaike, IEEE Trans. Autom. Control, 1974, 19, 716.

43 G. J. McLachlan and S. Rathnayake, WIREs Data Mining and Knowledge Discovery, 2014, 4, 341.

44 H. Häkkinen, Nat. Chem., 2012, 4, 443.

45 J. Turkevich, P. C. Stevenson and J. Hillier, J. Phys. Chem., 1953, 57, 670.

46 Z. Hens, I. Moreels and J. C. Martins, ChemPhysChem, 2005, 6, 2578.

47 H. D. Hill, J. E. Millstone, M. J. Banholzer and C. A. Mirkin, ACS Nano, 2009, 3, 418.

48 C. D. Walkey, J. B. Olsen, H. Guo, A. Emili and W. C. W. Chan, J. Am. Chem. Soc., 2012, 134, 2139.
49 K. Rahme, L. Chen, R. G. Hobbs, M. A. Morris, C. O'Driscoll and J. D. Holmes, RSC Adv., 2013, 3, 6085.

50 H.-M. Gao, H. Liu, H.-J. Qian, G.-S. Jiao and Z.-Y. Lu, Phys. Chem. Chem. Phys., 2018, 20, 1381.

51 B. Shi, C. Fang and Y. Pei, J. Pharm. Sci., 2006, 95, 1873.

52 S. Shen, H. Tang, X. Zhang, J. Ren, Z. Pang, D. Wang, H. Gao, Y. Qian, X. Jiang and W. Yang, Biomaterials, 2013, 34, 3150.

53 Y.-S. Chen, W. Frey, S. Kim, K. Homan, P. Kruizinga, K. Sokolov and S. Emelianov, Opt. Express, 2010, 18, 8867.

54 C. Wang, Z. Ma, T. Wang and Z. Su, Adv. Funct. Mater., 2006, 16, 1673.

55 E. Choi, M. Kwak, B. Jang and Y. Piao, Nanoscale, 2013, 5, 151.

56 W.-C. Wu and J. B. Tracy, Chem. Mater., 2015, 27, 2888.

57 M. Faria, M. Bjornmalm, K. J. Thurecht, S. J. Kent, R. G. Parton, M. Kavallaris, A. P. R. Johnston, J. J. Gooding, S. R. Corrie, B. J. Boyd, P. Thordarson, A. K. Whittaker, M. M. Stevens, C. A. Prestidge, C. J. H. Porter, W. J. Parak, T. P. Davis, E. J. Crampin and F. Caruso, Nat. Nanotechnol., 2018, 13, 777.

58 The code of multi-Lorentzian-splitting algorithm is available at: https:/github.com/Shirui816/MultipleDistributionFitting, The audiences with nonprofit objectives can cite this article to use the code for free. 